\title{
Diferenciación sexual en la langosta de agua dulce Cherax quadricarinatus (Decapoda: Parastacidae)
}

\author{
Fernanda J. Vazquez \& Laura S. López Greco* \\ Laboratorio de Fisiología Animal Comparada, Departamento de Biodiversidad y Biología Experimental, Facultad \\ de Ciencias Exactas y Naturales, Universidad de Buenos Aires, Ciudad Universitaria, C1428 EHA, Buenos Aires, \\ Argentina; laura@bg.fcen.uba.ar; fvazquez@bg.fcen.uba.ar \\ *Corresponding author
}

Recibido 26-X-2005. C Corregido 04-IV-2006. Aceptado 16-III-2007.

\begin{abstract}
Sexual differentiation in the freshwater crayfish Cherax quadricarinatus (Decapoda: Parastacidae). We determined the earliest stage of postembryonic development at which primary and secondary sexual differentiations occur in the freshwater ("red claw") crayfish Cherax quadricarinatus. For this purpose, 797 males, 506 females and 456 intersex specimens within a weight range of 0.02-89.96 g were observed under stereoscopic microscope to determine the presence of the genital openings at the basis of the third (females) or fifth (males) pair of pereiopods. Animals presenting both pairs of genital openings were considered as intersex. A subsample of 106 males, 69 females and 59 intersex were dissected for the macroscopic characterization of gonad morphology. The development of the genital openings began approximately at $0.10 \mathrm{~g}$, simultaneously with the differentiation of the reproductive system in females, males and intersex. Although the differentiation of the reproductive system started very early in the postembryonic development, the definitive form and colour of ovaries, oviducts, testes and vasa deferentia was acquired later. The differentiation of the appendix masculina began at 0.12-0.2 g and acquired the elongated shape of the adult at 1-2 g. The soft red patch characteristic of adult males started at $2.3 \mathrm{~g}$ of body weight in both chelipeds. Rev. Biol. Trop. 55 (Suppl. 1): 33-38. Epub 2007 June, 29.
\end{abstract}

Key words: Cherax quadricarinatus, sexual differentiation, primary sexual characters, secondary sexual characters, gonad, appendix masculina, red patch.

Cherax quadricarinatus (von Martens, 1868) ("red claw") es una langosta de agua dulce de la familia Parastacidae originaria de la región norte de Australia y el sur de Papua Nueva Guinea. Su aprovechamiento acuícola comenzó a fines de la década del 80 y continúa siendo una producción de tipo intensiva y semi-intensiva en expansión (Lawrence y Jones 2002). Es una especie de elevado precio en el mercado, rápido crecimiento, poca agresividad, sencilla reproducción, con buena fecundidad y desarrollo directo, que tiene rápida aceptación de los alimentos balanceados, elevada sobrevida fuera del agua, por lo que se puede comercializar viva manteniendo todas las propiedades de su carne, y ausencia de enfermedades de difícil erradicación. En Suramérica, se han desarrollado importantes proyectos en Ecuador y México (Lawrence y Jones 2002) siendo relativamente reciente su cultivo en Argentina, con interesantes perspectivas para los productores locales, tanto como alimento como para acuarismo (Luchini 2004, Panné Huidobro et al. 2004, Edgerton 2005).

Aunque se han investigado numerosos aspectos de la reproducción, aplicados al cultivo de C. quadricarinatus (Jones 1995, 1997, Sagi et al. 1996, 1997, Karplus et al. 2003, Vazquez et al. 2004), varias características de 
la biología reproductiva básica de la especie, con interesantes perspectivas de aplicación, aún no han sido dilucidadas. En particular, se desconoce el momento del desarrollo en que se produce la diferenciación sexual, tanto primaria (gonadal) como secundaria (aparición de los caracteres sexuales secundarios). Este conocimiento, permitiría identificar el momento más adecuado y temprano para separar a los ejemplares de distinto sexo, y así lograr la optimización del cultivo monosexo, dado que se ha demostrado que los machos crecen un 68 \% más rápido que las hembras, y que tanto los machos como las hembras en cultivo monosexo, crecen respectivamente un $17 \%$ y $31 \%$ más que en los cultivos mixtos (Curtis y Jones 1995). Por otra parte, la identificación temprana de las hembras podría permitir la inducción temprana de la reversión sexual, mediante factores ambientales y/o hormonales.

En función de la limitada información disponible sobre los aspectos anteriormente mencionados, el presente trabajo tiene por objetivo determinar el momento del desarrollo en que se produce la diferenciación de los caracteres sexuales de machos, hembras e intersexos en C. quadricarinatus.

\section{MATERIALES Y MÉTODOS}

Para determinar el momento del desarrollo en que se produce la diferenciación sexual se mantuvieron en laboratorio cuatro hembras ovígeras con embriones próximos a la eclosión (Jones 1995), obtenidas a partir de apareamientos logrados en laboratorio. Al producirse la eclosión de los juveniles I, se mantuvo a cada hembra con sus crías hasta el momento de la "independencia" de los mismos (estadio juvenil III, Levi et al. 1999).

Al independizarse todos los juveniles de cada hembra, los mismos fueron colocados en acuarios de $50 \times 40 \times 30 \mathrm{~cm}$ conteniendo $10 \mathrm{l}$ de agua corriente declorinizada, a $\mathrm{pH}$ 7.5-7.8, aireación constante, temperatura de $26-27{ }^{\circ} \mathrm{C}$, fotoperíodo de $14 \mathrm{~h}$ luz: $10 \mathrm{~h}$ oscuridad, alimentación ad libitum con Elodea sp. y alimento balanceado para peces disco Tetradiskus ${ }^{\circledR} \mathrm{y}$ un trozo de red tipo cebollera que los animales utilizaron como refugios. Semanalmente, se separaron al menos tres juveniles de cada hembra. Estos juveniles fueron pesados y sexados bajo lupa, en función de los caracteres sexuales secundarios. De este modo se obtuvieron juveniles desde un peso de $0.02 \mathrm{~g}$ (peso de los juveniles III al momento de la independencia) hasta los 8 g. También fueron analizados animales de peso mayor a $8 \mathrm{~g}$ provenientes de un criadero local. El total de animales examinados fue de 797 machos, 506 hembras y 456 intersexos.

Para evaluar la diferenciación sexual se sexaron bajo lupa los ejemplares, registrando la localización de los gonoporos (en la base del tercer par de pereiópodos para las hembras, y en la base del quinto par para los machos), la presencia o no de appendix masculina (ubicado en la base del quinto par de pereiópodos de los machos) y su correspondiente morfología y la presencia de la callosidad roja (“red patch”), característica de los machos de la especie, en el propodo del primer par de pereiópodos (Thorn y Fielder 1991). Dado que la especie presenta una proporción variable de individuos intersexos, se evaluó la presencia de éstos mediante la observación de los caracteres sexuales secundarios, considerándose intersexos a aquellos animales que presentaron gonoporos adicionales a los observados en la base del tercer ó quinto par de pereiópodos (Medley y Rouse 1993). Posteriormente, se tomó una muestra de 106 machos, 69 hembras y 59 intersexos que fueron anestesiados con frío y disecados bajo lupa, registrándose los aspectos macroscópicos de forma y color de la gónada.

\section{RESULTADOS}

Las observaciones sobre la diferenciación de los caracteres sexuales se indican a continuación, referidos al peso de los especímenes como estimación de su estado de desarrollo ontogenético. 


\section{Hembras}

Diferenciación de los gonoporos: a partir de los 0.08-0.09 g (en pesos menores no se distingue el sexo externamente).

Diferenciación ovárica macroscópica: entre los 0.09-0.5 g.

Coloración ovárica: la gónada presenta coloración transparente desde los 0.09 g hasta los 2.7 g, crema-pálido desde los 2.7 g hasta los 8.00-8.80 g, naranja desde los $8.10 \mathrm{~g}$ hasta los 12-15 g y adquiere la coloración verde oliva intensa, característica de los adultos de la especie, a partir de los $15 \mathrm{~g}$.

Morfología ovárica: las gónadas de color transparente, crema pálido y naranja, presentan forma de $\mathrm{H}$, con un par de lóbulos anteriores y un par posteriores, claramente diferenciados. La gónada verde oliva presenta cierto grado de fusión de los lóbulos posteriores tendiendo a adquirir forma de $\mathrm{Y}$.

\section{Machos}

Diferenciación de los gonoporos: desde los $0.10-0.11 \mathrm{~g}$ (en pesos menores no se distingue el sexo externamente).

Diferenciación y morfología del appendix masculina: el appendix masculina inicia su diferenciación a partir de los 0.12-0.2 g y presenta forma lobulada. Esta estructura comienza a elongarse y adquiere el patrón de estriación longitudinal característico de los machos adultos a partir de 1-2 g.

Diferenciación del "red patch": este carácter inicia su diferenciación entre los 2.3 a $2.8 \mathrm{~g}$ y de modo simultáneo en ambos quelípedos

Diferenciación testicular y del vaso deferente: el sistema reproductor se diferencia macroscópicamente a partir de los 0.1-0.2 g, identificándose claramente la diferencia entre testículo y vaso deferente a partir de 1.2-2 g. El vaso deferente se pliega y adquiere la coloración blanquecina, característica de los machos adultos (López et al. 2004), a partir de los 2-4 g, coincidiendo con el inicio de la formación del espermatóforo.

\section{Intersexos}

De los 59 animales disecados, 56 presentaron sistema reproductor femenino, aberturas genitales de ambos sexos y ausencia de appendix masculina lo que se corresponde con el tipo sexual denominado "intersexos hembra" (Vazquez y López Greco 2005). De los tres restantes, uno presentó sistema masculino bilateral, abertura genital de ambos sexos y appendix masculina bilateral y dos presentaron sistema masculino de un lado y femenino del otro, con aberturas genitales de ambos sexos y appendix masculina unilateral (del mismo lado en que se observó sistema reproductor masculino). La diferenciación de los gonoporos y del sistema reproductor ocurrió a partir de los 0.11-0.30 g en todos los intersexos.

\section{DISCUSIÓN}

El presente trabajo demuestra que la diferenciación de los caracteres sexuales primarios y secundarios de $C$. quadricarinatus ocurre de modo simultáneo y muy tempranamente, aproximadamente a partir de los $0.10 \mathrm{~g}$, lo que de acuerdo a nuestras observaciones previas (Vazquez et al. 2004) corresponde al estadio juvenil VI ó VII. La identificación temprana del sexo es una herramienta muy útil desde el punto de vista aplicado, pues permitiría la separación temprana para el cultivo monosexo así como para el desarrollo de técnicas de reversión sexual, mediante la manipulación de factores ambientales u hormonales. Este estudio no ha sido realizado en ninguna otra especie del género Cherax Erichson, 1846, ni en otro Parastacidae, por lo cual no podemos establecer relaciones comparativas con otras especies del género o familia. En la langosta Pontastacus leptodactylus leptodactylus (Eschscholtz, 1823) (Astacidae) se ha determinado que la diferenciación de los caracteres sexuales secundarios ocurre aproximadamente en el estadio VII (Payen 1973), lo que sería comparable con las evidencias obtenidas en el presente trabajo para C. quadricarinatus 
Desde el punto de vista de la morfología gonadal, fue interesante constatar que los ovarios de las hembras e intersexos hembras de C. quadricarinatus presentan forma de $\mathrm{H}$ durante la fase temprana de maduración y adoptan forma de $\mathrm{Y}$ en la fase más avanzada. De acuerdo a nuestras observaciones macroscópicas (que deben ser confirmadas histológicamente) se produciría una fusión de los lóbulos posteriores conforme avanza la maduración ovárica. El ovario maduro en forma de Y es característico de las langostas Astacidae y Cambaridae (Ando y Makioka 1998), mientras que en los Parastacidae sudamericanos, Parastacus brasiliensis (von Martens, 1869) (de Almeida y Buckup 1997), P. defosssus Faxon, 1898 (de Almeida y Buckup 1999) y P. varicosus, Faxon, 1898, (Rudolph et al. 2001) los ovarios están constituidos por dos lóbulos unidos longitudinalmente en toda su extensión, lo que difiere de lo observado en $C$. quadricarinatus.

\section{AGRADECIMIENTOS}

Este trabajo fue financiado por el Proyecto de Inicio de Carrera $N^{\circ} 4248-138$ de la Fundación Antorchas, el proyecto UBACYT X143 de la Universidad de Buenos Aires y por el Consejo Nacional de Investigaciones Científicas y Tecnológicas (CONICET, resolución $\mathrm{N}^{\circ}$ 1106/2004).

\section{RESUMEN}

Determinamos el momento del desarrollo postembrionario en que se produce la diferenciación sexual primaria en la langosta Cherax quadricarinatus. Esta es evidenciada por la presencia de las gónadas y sus respectivos conductos. También determinamos la diferenciación sexual definida por la aparición de los caracteres sexuales secundarios. Se observaron 797 machos, 506 hembras y 456 individuos intersexos de 0.02 a $89.96 \mathrm{~g}$ (de criadero y laboratorio). Disecamos una submuestra de 106 machos, 69 hembras y 59 individuos intersexos para la caracterización macroscópica de la estructura gonadal. La diferenciación de los gonoporos se inicia aproximadamente a los $0.10 \mathrm{~g}$, en sincronía con la diferenciación del sistema reproductor en machos, hembras e intersexos. La adquisición de la forma definitiva de ovario, oviducto, testículo y vaso deferente son posteriores. El appendix masculina inicia su diferenciación a los 0.12-0.2 g y adquiere los rasgos característicos del appendix de los adultos a partir de 1-2 g. La diferenciación de la mancha roja ("red patch") ocurre a partir de los $2.3 \mathrm{~g}$.

Palabras clave: Cherax quadricarinatus, diferenciación sexual, caracteres sexuales primarios, caracteres sexuales secundarios, gónada, appendix masculina, “red patch”.

\section{REFERENCIAS}

Ando, H. \& T. Makioka. 1998. Structure of the ovary and mode of oogenesis in a freshwater crayfish, Procambarus clarkii (Girard). Zool. Sci. 15: 893901.

Curtis, M.C. \& C.M. Jones. 1995. Observations on monosex culture of redclaw crayfish Cherax quadricarinatus von Martens (Decapoda: Parastacidae) in earthen ponds. J. World Aquacult. Soc. 26: 154-158.

de Almeida, A. \& L. Buckup. 1997. Aspectos anatómicos e funcionais do aparelho reproductor de Parastacus brasiliensis (von Martens) (Crustacea, Decapoda, Parastacidae). Rev. Bras. Zool. 14: 497-509.

de Almeida, A. \& L. Buckup. 1999. Caracteres sexuais primaries e secundarios do lagostim Parastacus defossus Faxon, 1898 (Crustacea, Parastacidae). Nauplius 7: 113-126.

Edgerton, B.F. 2005. Freshwater crayfish production for poverty alleviation. World Aquacult. 36: 48-64.

Jones, C.M. 1995. Production of juvenile redclaw crayfish, Cherax quadricarinatus (von Martens) (Decapoda, Parastacidae). I. Development of hatchery and nursery procedures. Aquaculture 138: 239-245.

Jones, C.M. 1997. The biology and aquaculture potential of the tropical freshwater crayfish Cherax quadricarinatus. Department of Primary Industries, Queensland Government, Queensland, Australia. $130 \mathrm{p}$.

Karplus, I., H. Gideon \& A. Barki. 2003. Shifting the natural spring-summer breeding season of the Australian freshwater crayfish Cherax quadricarinatus into the winter by environmental manipulations. Aquaculture 220: $277-286$.

Lawrence, C. \& C. Jones. 2002. Cherax, p. 635-669. In D. Holdrich (ed.). Biology of Freshwater Crayfish, 
Blackwell Science, Malden, Massachusetts, EEUU.

Levi, T., A. Barki, G. Hulata \& I. Karplus. 1999. Mother-offspring relationships in the red-claw crayfish (Cherax quadricarinatus). J. Crust. Biol.19: 477-484.

López Greco, L.S., A. Chaulet, F.J. Vazquez \& E.M. Rodríguez. 2004. Morphology of testes, vasa deferentia and spermatophores in the freshwater crayfish, Cherax quadricarinatus (Parastacidae). Proceedings of the $3^{\text {rd }}$ Brazilian Crustacean Congress and 2004 TCS Summer Meeting, Santinho, Florianópolis, Brasil, 24-28 de octubre. p. 210.

Luchini, L. 2004. Perspectivas en acuicultura, nivel mundial, regional y local. Secretaría de Agricultura, Ganadería, Pesca y Alimentos (SAGPyA), Subsecretaría de Pesca y Acuicultura, Dirección de Acuicultura, Buenos Aires, Argentina. 96 p.

Medley P., \& D.B. Rouse. 1993. Intersex Australian red claw crayfish (Cherax quadricarinatus). J. Shellfish. Res. 12: 93-94.

Payen, G. 1973. Etude descriptive des principales étapes de la morphogenésis sexuelle chez un crustacé décapode a développement condensé, I'écrevisse Pontastacus leptodactylus leptodactylus (Eschscholtz, 1823). Ann. Embryol. Morphol. 6: 179-206.

Rudolph, E.H., A. Verdi \& J. Tapia. 2001. Intersexuality in the burrowing crayfish Parastacus varicosus Faxon, 1898 (Decapoda, Parastacidae). Crustaceana 74: 27-37.

Sagi, A, I. Khalaila, A. Barki, G. Hulata \& I. Karplus. 1996. Intersex red claw crayfish Cherax quadricarinatus (von Martens): functional males with pre-vitellogenic ovaries. Biol. Bull. 190: 16-23.
Sagi, A, A. Milstein, Y. Eran, D. Joseph, I. Khalaila, U. Abdu, S. Harpaz \& I. Karplus. 1997. Culture of the Australian redclaw crayfish (Cherax quadricarinatus) in Israel. Second growthout season of overwintered populations. Israeli J. Aquacult. 49: 222-229.

Thorn, M.J. \& D.R. Fielder. 1991. The red cuticle on the claw of male Cherax quadricarinatus (Decapoda: Parastacidae). Mem. Queensl. Mus. 31: 277.

\section{REFERENCIAS DE INTERNET}

Panné Huidobro, S., M. Alvarez \& L. Luchini. 2004. Aspectos de la comercialización de peces ornamentales en Argentina (Importación y Exportación, Período 1999-2003). Secretaría de Agricultura, Ganadería, Pesca y Alimentos (SAGPyA), Subsecretaría de Pesca y Acuicultura, Dirección de Acuicultura, Buenos Aires, Argentina. (Consultado: 10 de mayo 2006, http://www.sagpya.mecon.gov.ar/new/0-0/ pesca/acuicultura/ornamentales/ornamentales.pdf ).

Vazquez, F. \& L. López Greco. 2005. Intersexos hembras en la langosta de agua dulce Cherax quadricarinatus (Decapoda, Parastacidae). Resúmenes del XI Congreso Latinoamericano de Ciencias del Mar, Viña del Mar, Chile, 16-20 de mayo. (Consultado: 10 de julio 2005, http://www.colacmar.cl/ 83-86).

Vazquez. F.J, A. Chaulet, A. Bugnot \& L.S. López Greco. 2004. Efecto de la temperatura sobre la diferenciación sexual en juveniles de la langosta de agua dulce, Cherax quadricarinatus (Astacida, Parastacidae). III Congreso Iberoamericano Virtual de Acuicultura, 19 de noviembre-19 de diciembre. (Consultado: 10 de mayo 2006, http://www.civa2004.org). 
